\title{
Imaging Techniques for Small Animal Models of Pulmonary Disease: MR Microscopy
}

\author{
Bastiaan Driehuys and Laurence W. Hedlund \\ Center for In Vivo Microscopy, Duke University Medical Center, Durham, North Carolina 27710, \\ USA
}

\section{Abstract}

In vivo magnetic resonance microscopy (MRM) of the small animal lung has become a valuable research tool, especially for preclinical studies. MRM offers a noninvasive and nondestructive tool for imaging small animals longitudinally and at high spatial resolution. We summarize some of the technical and biologic problems and solutions associated with imaging the small animal lung and describe several important pulmonary disease applications. A major advantage of MR is direct imaging of the gas spaces of the lung using breathable gases such as helium and xenon. When polarized, these gases become rich MR signal sources. In animals breathing hyperpolarized helium, the dynamics of gas distribution can be followed and airway constrictions and obstructions can be detected. Diffusion coefficients of helium can be calculated from diffusion-sensitive images, which can reveal micro-structural changes in the lungs associated with pathologies such as emphysema and fibrosis. Unlike helium, xenon in the lung is absorbed by blood and exhibits different frequencies in gas, tissue, or erythrocytes. Thus, with MR imaging, the movement of xenon gas can be tracked through pulmonary compartments to detect defects of gas transfer. MRM has become a valuable tool for studying morphologic and functional changes in small animal models of lung diseases.

\section{Keywords}

MRI; hyperpolarized; ${ }^{3} \mathrm{He} ;{ }^{129} \mathrm{Xe}$; lung imaging; pulmonary function; rodent

\section{Introduction}

To study the function of rodent lungs, one might like to directly image the flow of gases into the lungs, the gas exchange across the blood-gas barrier, and to see gases carried away by the pulmonary circulation. Additionally, one might like to wrap this detailed functional picture in a high-quality anatomical image. Finally, these techniques should be noninvasive so that specific animals can be monitored over time as they either develop disease or respond to a specific course of treatment. With advances in magnetic resonance microscopy, the goal of morphologic imaging has been largely met (Hedlund and Johnson, 2005).

MR imaging methods and applications to small animal models of lung disease will be briefly reviewed here. Perhaps the most significant problem associated with imaging the lung is imaging the gas spaces that account for up to $80 \%$ of its volume and provide no intrinsic signal. Of any imaging modality, MR provides a unique solution to airway imaging by using special breathable gases that are MR signal sources. The development of hyperpolarized noble gases for MR imaging has been a major accomplishment in the past decade, making it possible to

Address correspondence to: Bastiaan Driehuys, Center for In Vivo Microscopy, Box 3302, Duke University Medical Center, Durham, NC 27710, USA; driehuys@ orion.duhs.duke.edu. 
image their distribution with exquisite resolution despite their 3000-fold lower density than tissue.

Gas imaging has been realized through an ongoing convergence of atomic physics research, biomedical engineering, and magnetic resonance imaging (MRI) research. Combined structural and functional MRI of the lung is thus poised to become a powerful tool in small animal research of the respiratory system. Thus, we will also provide a detailed overview of hyperpolarized gas MRI and the functional information that can be derived from this technology with a focus on application to small animal models of pulmonary disease.

\section{MRI Basics}

To appreciate the special challenges and opportunities of MR microscopy in small animals, it is instructive to briefly review the basic operating principles of MRI (Bushberg et al., 2001; Hornak, 2006). The signal source in conventional MRI is water; more specifically, the tiny magnetic moments of the protons that make up the nuclei of the hydrogen atoms in water. To derive a signal from protons requires them to oscillate, thereby changing the magnetic flux they present to a surrounding receiver coil (a loop of wire). A changing magnetic flux in a coil induces a voltage just as the rotating turbine of a power plant spins a large magnet that induces the familiar $60 \mathrm{~Hz}$ voltage coming out of our walls. Protons (or any magnetic moments) can be enticed to oscillate by placing them in a magnetic field and tickling them with a radio frequency pulse. In a magnetic field, protons can either line up with the magnetic field or against it.

A radiofrequency pulse applied to the protons via the same coil used for detection, torques them away from the magnetic field axis and causes them to wobble around this axis at a frequency that is precisely proportional to the strength of the magnetic field and their own magnetic moment. This wobbling is called precession. For example, in a clinical 1.5 Tesla MRI scanner, the ${ }^{1} \mathrm{H}$ precession frequency is about $64 \mathrm{MHz}$. The flux from the spinning nuclear moments is picked up by the coil, amplified, and digitized. In the case of water protons, after a few hundred milliseconds of precessing, the protons return back to being aligned with the magnetic field and they can be pulsed and detected again.

To generate a magnetic resonance image rather than a single-frequency signal, we apply a welldefined, linear magnetic field gradient during signal acquisition. The gradient reduces the magnetic field strength at one end of the sample and increases it at the other end. This makes the nuclei at one end of the sample precess more slowly than at the other because precession frequency is proportional to magnetic field strength. The detected signal is then broken down by its frequency components whose amplitudes represent a 1-dimensional image of the sample. This approach of using magnetic field gradients to encode the spatial distribution of nuclei can be readily extended to acquire images in 3 dimensions.

The final role of the magnetic field in imaging is to bring enough order to the system of nuclei to make them detectable. To see an MRI signal, an imbalance must exist in the number of nuclei starting out aligned versus anti-aligned with the magnetic field. These two populations have precession signals of opposite signs and would cancel each other exactly if present in equal numbers. Fortunately, the interaction of the nuclei with the magnetic field makes it slightly more energetically favorable for them to be aligned rather than anti-aligned with the field. This imbalance in the population of nuclei is called polarization and is defined as $P=1$ $N_{\uparrow}-N_{\downarrow} \mid /\left(N_{\uparrow}+N_{\downarrow},\right)$ where $N_{\uparrow}$ is the number of aligned nuclei and $N_{\downarrow}$ is the number of antialigned nuclei. However, even in large magnetic fields such as that of a 1.5 Tesla clinical MRI scanner, the tiny energy difference between states gives a polarization only 5 parts per million. Thus, most of the nuclei still cancel one another. 
The strength of the MRI signal coming from a given three-dimensional pixel element (voxel) in the image is

$$
S_{\mathrm{MRI}} \alpha \mu \times \omega \times P \times \rho \times V_{\mathrm{vox}}
$$

where $\mu$ is the nuclear magnetic moments, $\omega$ is their precession frequency around the magnetic field, $P$ is their polarization, $\rho$ is their density, and $V_{v o x}$ is the voxel volume. Both precession frequency $\omega$ and polarization $P$ increase linearly with magnetic field strength that drives the neverending quest for higher magnetic field strengths in MRI. The intuitive dependence on density also explains why water is the favored imaging source. The density of hydrogen nuclei $\left[{ }^{1} \mathrm{H}\right]$ is $110 \mathrm{M}$, sufficient to provide ample signal despite relatively low nuclear polarization in even the largest magnetic fields.

\section{Proton MR Microscopy for Morphology}

As one translates proton MR imaging from clinical scales to the microscopic scales required for rodents, numerous additional challenges emerge. To image a human chest, we might take a field of view of $36 \times 36 \mathrm{~cm}$ and image it in $10 \mathrm{~mm}$-thick slices with an in-plane resolution of $256 \times 256$. Thus, a typical voxel in such an image is $1.4 \times 1.4 \times 10 \mathrm{~mm}^{3}$, comprising a volume of $\sim 20 \mu \mathrm{L}$. To resolve comparable structures in a mouse chest, we employ a field of view of about $2 \mathrm{~cm}$, and use $0.1 \mathrm{~mm}$ slices resulting in voxels of $78 \times 78 \times 100 \mu \mathrm{m}^{3}$, each with a volume of only $0.6 \mathrm{~nL}$. Equation (1) tells us that the signal in a mouse voxel will be 33,000 times smaller than in the human. These signal deficits can be partially compensated by designing more efficient receiver coils, but the remainder must be made up through signal averaging. However, acquiring images over many respiratory and cardiac cycles introduces undesired motion artifacts, if not controlled.

Rodent respiratory and cardiac rates are up to an order of magnitude faster than in human subjects and animals cannot be instructed to hold their breath during imaging. Constant motion can degrade image quality with blurring and artifacts, so animals are placed on an MRcompatible ventilator (Hedlund et al., 2000; Mai et al., 2005) that controls the breathing cycle. Positioning of the lung is precisely reproduced from breath to breath (Mai et al., 2005) as the MR-compatible ventilator triggers the MR scanner to acquire data only during a specified phase of the breathing cycle, such as at end-expiration or at full inspiratory volume. Heart motion can also be frozen by providing a second image gating signal synchronized with the cardiac cycle (Brau et al., 2004). Additionally, to achieve resolution on the order of $100 \mu \mathrm{m}$ requires large magnetic field gradients to create the needed frequency dispersion to distinguish neighboring voxels of such small dimensions. Thus, dedicated animal scanners with gradients an order of magnitude stronger than their clinical counterparts are a necessity for MRM.

Finally, of all organs, the lung presents the biggest challenge for MR imaging. First, its low tissue density compared to other organs makes it an unusually poor source of proton signal. Furthermore, the very structure of the lung with its myriad of gas/alveolar tissue interfaces causes the MR signal to decay more rapidly than occurs in solid tissues. This further reduces the already low signal intensity in the lung. To overcome this problem, specialized imaging sequences have been developed to capture signal more rapidly, within microseconds instead of the milliseconds used in conventional scans (Gewalt et al., 1993).

The apparatus used in a typical ventilation-controlled imaging session of a mouse is show in Figure 1 . The mouse is intubated with a 20 -gauge endotracheal tube and ventilated with an MR-compatible constant volume ventilator at 100 breaths per minute with $0.2 \mathrm{ml}$ tidal volume (Chen et al., 2003). The imaging coil is dual-tuned to support hyperpolarized ${ }^{3} \mathrm{He}$ and ${ }^{1} \mathrm{H}$ 
imaging on a 2 T MR scanner. Airway pressure, temperature, and ECG are monitored continuously and body temperature is controlled by warm air circulating through the bore of the magnet using feedback from a rectal temperature probe.

The ventilator triggers the MRI scanner at end inspiration or end expiration to capture image data during this brief moment when lung motion is suspended. Images are acquired over multiple breaths to attain sufficient signal-to-noise in the nanoliter voxels, typically 20-40 acquisitions per breath, until the necessary number of image frames has been acquired. To achieve the short echo times necessary for lung imaging, we employ radial sampling of the image space, which requires us to acquire 800 "rays" of image data to adequately sample a single image "slice" with a $256 \times 256$ matrix. Thus, we require roughly 40 well-controlled breath-hold periods to acquire one image slice. Extending to high-resolution 3D imaging can easily expand the required number of image views to 100,000 or more, which can take a good fraction of an hour to acquire. Such calculations illustrate the critical interplay of image acquisition requirements with the need for precise ventilatory control over the animal. Figure 2 shows an example of a proton morphologic image of a rat lung acquired in this manner with an isotropic resolution of $117 \times 117 \times 117 \mu \mathrm{m}^{3}$ (Johnson et al., 2001).

This exquisite capability of proton MR to reveal soft tissue differences and its sensitivity to tissue water has made MR a valuable tool to study pulmonary pathologies associated with edema, inflammation, fibrosis, and emphysema. For instance, in a model of edema and fibrosis lung injury from the herbicide paraquat, animals were followed with MR microscopy (MRM) from day 1 of exposure with development of extensive edema, to its resolution after 7 days, and development of fibrosis by day 14 in rats (Hedlund et al., 1996). Rats exposed to hyperoxia $(85 \%)$ for 7 days exhibited on day 1 peribronchial inflammation, on day 4 peribronchial edema and severe pleural effusion, more severe edema by day 5 , while effusions were slightly diminished, and by day 7, the edema and effusion were completely reabsorbed (Hedlund et al., 1996). Small animal MR imaging has also been used to study pulmonary edema resulting from allergen challenge in rats (Beckmann et al., 2001).

\section{Hyperpolarized Gas MRI Basics}

One of the most important features of pulmonary MRI is the capability to directly image the airway structure of the lung using inhaled gases. However, to image gases using MRI requires us to overcome substantial obstacles, since the low density would normally render the gas invisible. Hyperpolarization is the technical trick that makes it possible to image the gaseous stable isotopes ${ }^{3} \mathrm{He}$ and ${ }^{129} \mathrm{Xe}$ with the same sort of exquisite spatial resolution that we have come to expect from "conventional" proton MRI. Hyperpolarized ${ }^{3} \mathrm{He}$ and ${ }^{129} \mathrm{Xe}$ have their nuclei in nearly perfect alignment with the magnetic field as compared to the relatively weak alignment generated by thermal equilibrium dynamics. As can be seen in Equation (1) with polarization levels approaching $50 \%$ rather than $0.0005 \%$, the signal enhancement of roughly $10^{6}$ is more than sufficient to overcome the density deficit that gases suffer compared to solid tissues.

Hyperpolarization of ${ }^{3} \mathrm{He}$ and ${ }^{129} \mathrm{Xe}$ has been an active field of physics research for several decades, but the application of these gases to biological imaging has spurred renewed activity and fairly dramatic leaps in the technology over the past decade. Excellent introductory references are given by Leawoods et al. (2001), Moller et al. (2002), and Kadlecek (2002). The essence of the hyperpolarization relies on angular momentum transfer from light. The difference between a nuclear moment precessing clockwise vs. counterclockwise around the magnetic field is exactly one quantum of angular momentum. By adding enough angular momentum to an ensemble of nuclei, the nuclei become polarized. 
A ready supply of angular momentum is carried by photons, which when circularly polarized, each carry +1 quantum of angular momentum. Since nuclei cannot directly absorb light, an intermediary is needed to absorb the light (and the angular momentum). Circularly polarized laser light is absorbed by an alkali metal atom such as rubidium $(\mathrm{Rb})$, causing the outer valence electron of each atom to precess in the same direction around the local magnetic field (electron spin polarization). Subsequent collisions between the polarized valence electrons and the noble gas nuclei slowly exchange the angular momentum stored in electron spins to angular momentum stored in nuclear spins of the gas. This method of three-step angular momentum transfer is known as optical pumping and spin exchange as illustrated in Figure 3.

While hyperpolarization physics is elegant and complex, the practical implementation of the method is more straightforward. To an outside observer, hyperpolarization appears merely as shining a bright laser on a glass container holding a blob of $\mathrm{Rb}$ alkali metal and a substantial density of ${ }^{3} \mathrm{He}$ or ${ }^{129} \mathrm{Xe}$ gas. The gas is contained under pressure ( $\left.\sim 10 \mathrm{~atm}\right)$ in an optical cell and heated to about $200^{\circ} \mathrm{C}$ to create a dilute vapor $\left(\sim 10^{-6} \mathrm{~atm}\right)$ of $\mathrm{Rb}$ to absorb the laser light. Once the nuclear polarization is saturated, it is stabilized in the ${ }^{3} \mathrm{He}$ or ${ }^{129} \mathrm{Xe}$ nuclei by cooling the system down so that all $\mathrm{Rb}$ atoms condense out of the vapor phase and spin exchange stops. A schematic of such a system is shown in Figure 4.

Typical production rates are $\sim 0.5-1$ liter per hour of polarized ${ }^{3} \mathrm{He}$ or ${ }^{129} \mathrm{Xe}$. Such quantities of gas are sufficient for imaging human lung function in a single breath, or imaging rodent lung function over many breaths. Moreover, if the hyperpolarized gas is maintained in an environment free of magnetic impurities, it retains its nuclear polarization for hours or even days, thus allowing the gases to be readily transported long distances from the polarizer to the MR scanner (van Beek et al., 2003). An important consequence of hyperpolarization versus thermal equilibrium polarization employed for ${ }^{1} \mathrm{H}$ MRI is that since ${ }^{1} \mathrm{H}$ is polarized by the magnetic field, it can be re-polarized by the same field, whereas ${ }^{3} \mathrm{He}$ and ${ }^{129} \mathrm{Xe}$ being polarized by light are not re-polarized in the magnetic field and become completely silent after imaging. Since the very act of imaging the gas causes it to lose its hyperpolarized state, scan protocols must be specially tailored to extract maximum information from a single dose of hyperpolarized gas. However, selective destruction of the hyperpolarized state of ${ }^{129} \mathrm{Xe}$ can also be used to advantage in following gas transfer in the lung (described in the next sections).

\section{Airspace MRM with Hyperpolarized ${ }^{3} \mathrm{He}$}

Biological imaging of hyperpolarized gases dates back to the first visualization of ${ }^{129} \mathrm{Xe}$ in a mouse lung preparation by Albert and co-workers in 1994. Since that demonstration, the field moved quickly to in vivo small animal imaging (Black et al., 1996) and the first demonstrations in human subjects (Ebert et al., 1996; MacFall et al., 1996). In the clinical realm, hyperpolarized ${ }^{3} \mathrm{He}$ MRI of airspace ventilation has been applied to study asthma (Samee et al., 2001, 2003), chronic obstructive pulmonary disease (COPD) (Salerno et al., 2002; Fain et al., 2006), cystic fibrosis (Donnelly et al., 1999; Koumellis et al., 2005; Mentore et al., 2005), and bronciolitis obliterans associated with lung transplant rejection (McAdams et al., 1999).

As with ${ }^{1} \mathrm{H}$ MR imaging where many forms of soft tissue contrast can be exploited such as $T_{1}, T_{2}$, or diffusion-weighted imaging, hyperpolarized gas imaging can be readily extended beyond simple "gas density" imaging. Additional MR contrast mechanisms for hyperpolarized ${ }^{3} \mathrm{He}$ include apparent diffusion coefficient (ADC) imaging for assessing alveolar microstructure (Chen et al., 2000; Salerno et al., 2002), dynamic imaging of gas inflow (Salerno et al., 2001a; Viallon et al., 2000), and regional measurement of oxygen partial pressure (Deninger et al., 1999; Kadlecek et al., 2005). Excellent reviews on the clinical exploration of hyperpolarized ${ }^{3} \mathrm{He}$ can be found in (Salerno et al., 2001b; van Beek et al., 2004). 
Small animal imaging of hyperpolarized gases is predictably more challenging than clinical imaging. After all, the human patient can be asked to take a deep breath of gas from a bag and hold for 10 or 15 seconds while imaging is performed, but not so with a rodent. In addition to motion degradation of image quality previously discussed for ${ }^{1} \mathrm{H} \mathrm{MRM}$, blurring and attenuation can occur because of the high diffusivity of ${ }^{3} \mathrm{He}$ gas, creating an additional obstacle for resolving small airways and related structures. Thus, specialized MRI image acquisition strategies (pulse sequences) have been implemented specifically for high-quality imaging of hyperpolarized gases in small animals (Johnson et al., 1997).

These techniques have been used to study models of pulmonary pathology in the rat and when used with a dual-tuned imaging coil, both solid tissue (proton) and gas distribution $\left({ }^{3} \mathrm{He}\right)$ can be obtained in the same imaging session. Furthermore, imaging hyperpolarized helium at several different gradient strengths yields data that can be used to calculate an ADC, which can reveal the underlying microstructure of the gas spaces of the lungs. For instance, rats treated with porcine elastase develop panacinar emphysema that results in a larger helium ADC because its diffusion is less restricted than in the normal lung (Chen et al., 2000).

Conversely, rat lungs exposed to therapeutic radiation develop interstitial fibrosis that reduces alveolar volumes, greatly restricting helium diffusion and resulting in a lower ADC than in a normal lung (Ward et al., 2004). In these examples, concurrent proton images reveal the underlying structural changes and associated reduction in parenchymal signal intensity seen in the emphysema model and elevated signal intensities in the fibrotic lung.

Although imaging the rat lung with HP gas and proton MR is challenging, an even greater challenge is to image the mouse lung. However, the availability of genetically manipulated mouse models for research on pulmonary diseases like asthma and COPD has compelled researchers to develop techniques to image these creatures that are roughly one-tenth the size of a rat. Imaging allows us to better evaluate pulmonary function on a regional level in response to challenges and in response to therapeutic agents. The reduction of ${ }^{3} \mathrm{He}$ MRI to the scale of a mouse lung has proven difficult due to the small tidal volumes and the exquisite resolution needed to resolve airways of the mouse. At $\sim 200 \mu$ l, the tidal volume of the mouse is an order of magnitude smaller yet, than that of the rat. Hyperpolarized ${ }^{3} \mathrm{He}$ imaging in the mouse lung was first demonstrated by Dugas et al. (2004), who achieved a resolution of $0.25 \times 0.25 \times$ $3 \mathrm{~mm}^{3}$. Chen et al. (2005) demonstrated rather spectacular resolution images of ${ }^{3} \mathrm{He}$ distribution in mouse lungs with a resolution of $70 \times 70 \times 800 \mu \mathrm{m}^{3}$, but to do so, the animal consumed 1.2 liters of ${ }^{3} \mathrm{He}$ in 25 minutes of image acquisition time. Here, we will discuss our recent progress in creating high-resolution images in mouse models of asthma using modest quantities of hyperpolarized ${ }^{3} \mathrm{He}$ and sufficiently rapid scan times to efficiently image the transient narrowing of the airways induced by methacholine. Recently, we were able to observe the dramatic airway narrowing and reduced ${ }^{3} \mathrm{He}$ ventilation in BALB/C mice that had been sensitized with ovalbumin and challenged with methacholine (Driehuys et al., 2006b).

Hyperpolarized gas imaging takes advantage of the same scan-synchronous ventilator technology developed for controlling respiratory motion in ${ }^{1} \mathrm{H}$ structural MRI (Figure 1). A critical requirement is the ability to deliver hyperpolarized ${ }^{3} \mathrm{He}$ to the animal in microliter amounts at specific phases of breathing and in such a way as to prevent depolarization before reaching the lungs. This is accomplished by rapid remote control switching of the breathing gas from air to a mixture of $75 \% \mathrm{HP}^{3} \mathrm{He}$ mixed with $25 \% \mathrm{O}_{2}$ to achieve the $0.2 \mathrm{ml}$ tidal volume. Again, images are acquired over multiple breaths to achieve sufficient signal-to-noise (SNR) for the extremely high resolution employed. To reduce the effects of diffusion blurring, we use a radial sampling of the image space. We acquire 800 "rays" of image data to adequately sample a single image "slice" with a $256 \times 256$ matrix. Thus, we require roughly 40 well-controlled breaths to acquire one image slice in this manner. 
Figure 5 shows an example of a 3D ${ }^{3} \mathrm{He}$ image acquisition in a control mouse. The resolution of this image is $125 \times 125 \times 1000 \mu \mathrm{m}^{3}$, which required 5.8 minutes to acquire 11520 rays and consumed $92 \mathrm{ml}$ of ${ }^{3} \mathrm{He}$. This scan time is fast enough to capture the short duration airway responses from an intravenous injection of a muscarinic receptor agonist such as methacholine (Driehuys et al., 2006b). The use of ${ }^{3} \mathrm{He}$ MRI in imaging of mouse models of asthma provides a level of regional specificity that has never before been possible. The $3 \mathrm{D}$ images can be nicely represented as a maximum intensity projection (MIP) images to highlight the structure of the major airways, as shown in Figure 6. It is possible to acquire images even faster by acquiring a 2-dimensional projection of the whole lung, as also shown in Figure 6c. While the detail in this image is not as exquisite as that of the 3D composite, the image was acquired in only 12 seconds and consumed only $2 \mathrm{ml}$ of ${ }^{3} \mathrm{He}$. Thus, we see a wide range of possibilities for ${ }^{3} \mathrm{He}$ imaging to answer many biological questions. Although exquisite resolution requires significant time and gas consumption, it enables us to observe subtle functional changes not detectable by other means.

\section{Functional MRM with Hyperpolarized ${ }^{129} \mathrm{Xe}$}

While hyperpolarized ${ }^{3} \mathrm{He}$ has ushered in a capability to trace the morphology of the lung airspaces, hyperpolarized ${ }^{129} \mathrm{Xe}$ is poised to provide an even more complete picture of lung function by supplying information about the gas exchange process at the alveolar level. Unlike ${ }^{3} \mathrm{He}$, which is nearly insoluble in most tissues and fluids, ${ }^{129} \mathrm{Xe}$ has significant solubility in blood and all tissues in the body. Thus, inhaled ${ }^{129} \mathrm{Xe}$ is readily distributed throughout the body. More importantly, ${ }^{129} \mathrm{Xe}$ shifts its resonant frequency dramatically upon experiencing a new molecular environment. So, not only can one image ${ }^{129} \mathrm{Xe}$ distribution throughout all perfused organs, but one can discriminate the type of tissue environment in which it resides. This sensitivity to molecular environment results from the transient binding that xenon undergoes with almost all proteins (Cherubini and Bifone, 2003). Such interactions perturb its large electron cloud, which slightly alters the magnetic field experienced by the nucleus. This potentially powerful aspect of hyperpolarized ${ }^{129} \mathrm{Xe}$ has been overshadowed by its weaker MRI signal and less mature state of hyperpolarization technology than ${ }^{3} \mathrm{He}$.

Recent technical advancements have greatly improved ${ }^{129} \mathrm{Xe}$ imaging. Figure 7 shows a progression of small animal ${ }^{129} \mathrm{Xe}$ ventilation images from this laboratory, starting with the earliest work in 1998 (Chen et al., 1999) to images acquired today. Through a combined focus on the physics of hyperpolarization and MR imaging strategies, the image resolution we attain has improved by a factor of 7, while signal-to-noise has improved 3-fold. With these steps, ${ }^{129} \mathrm{Xe}$ is poised to start providing airspace images that are competitive with ${ }^{3} \mathrm{He}$. Moreover, such signal improvements now put us in a position to exploit the properties that make ${ }^{129} \mathrm{Xe}$ so interesting in the first place-solubility and chemical frequency shift.

A good example of exploiting the solubility and frequency shift of ${ }^{129} \mathrm{Xe}$ for deriving unique pulmonary function information can be seen in our study of pulmonary fibrosis (Driehuys et al., 2006a). When ${ }^{129} \mathrm{Xe}$ is inhaled into the lung and enters into the alveolar air-spaces, a small fraction is absorbed into the moist epithelial surface. ${ }^{129} \mathrm{Xe}$ dissolved in the alveolar epithelium, interstitium, capillary endothelium, and plasma resonates at a frequency that is shifted 197 parts per million (ppm) (4.64 kHz in a $2 \mathrm{~T}$ field) from the $23.64 \mathrm{MHz}$ gas reference frequency 0 ppm (Sakai et al., 1996). The atoms diffuse across the tissue barrier and their concentration equilibrates in the red blood cells (RBCs) where the resonant frequency shifts again to 211 ppm away from the gas resonance (Albert et al., 1995). Figure 8 illustrates this simple threecompartment model of the lung and the associated ${ }^{129} \mathrm{Xe}$ frequency spectrum. The ability to discriminate ${ }^{129} \mathrm{Xe}$ in these three compartments allows us to image gas transfer dynamics with unprecedented precision. In healthy lung, the barrier between airspace and RBC is only about $1 \mu \mathrm{m}$, a distance ${ }^{129} \mathrm{Xe}$ can diffuse across in just over a millisecond. However, if inflammation 
or fibrosis causes a thickening of this barrier to even $5 \mu \mathrm{m}$, the time for ${ }^{129} \mathrm{Xe}$ to diffuse across it and reach an RBC increases to $40 \mathrm{~ms}$. By imaging ${ }^{129} \mathrm{Xe}$ in the barrier and $\mathrm{RBC}$ with a radiofrequency pulse repetition rate on this time scale, dissolved ${ }^{129} \mathrm{Xe}$ is continually depolarized and becomes silent, only to be replenished by diffusion of fresh ${ }^{129} \mathrm{Xe}$ from the airspaces. Barrier thickening is readily identified by an absence of RBC signal in the images, which results from the inability of ${ }^{129} \mathrm{Xe}$ to diffusively traverse the barrier on the repetition timescale of the image.

With recent advancements in image quality and ${ }^{129} \mathrm{Xe}$ polarization and a novel imaging sequence, we have been able to directly image this process of alveolar-capillary gas transfer (Driehuys et al., 2006a). Figure 9 shows an image of ${ }^{129} \mathrm{Xe}$ in the airspace, barrier space, and red blood cells in a control rat and one with left-lung pulmonary fibrosis induced by intratracheal instillation of bleomycin. Note that in the left fibrotic lung, virtually no RBC signal can be detected, while the barrier image matches the ventilation pattern. The absence of signal in the RBCs is the result of the increased time it takes for ${ }^{129} \mathrm{Xe}$ to diffuse across the thickened blood-gas barrier, as predicted. Indeed, examination of the left lung via conventional histology after imaging showed significant thickening of the alveolar septa. This example illustrates how the ability to discriminate ${ }^{129} \mathrm{Xe}$ in different tissue compartments can lead to very powerful markers of function, in this case, gas transfer.

Further development of hyperpolarized ${ }^{129}$ Xe polarization technology and imaging strategies should lead to greatly improved images of both the airspace and ${ }^{129} \mathrm{Xe}$-containing tissue compartments. Combining the signal of hyperpolarized ${ }^{129} \mathrm{Xe}$ with additional agents such as cryptophane cages, which can be designed to bind to receptors of choice (Driehuys, 2006; Schroder et al., 2006), may in fact open up a brand new approach to molecular imaging of cellular processes.

\section{Discussion and Conclusion}

In the last few years, small animal imaging has become an increasingly important research tool for studying disease models (Schuster et al., 2004) and for preclinical testing (Beckmann et al., 2003). This increase in popularity of small animal imaging has been fostered partly by the ready availability of commercial imaging systems and the demonstration of the value of in vivo, nondestructive, and longitudinal monitoring capability of these imaging systems. MR microscopy, an outgrowth of clinical MRI, has a more than 20-year history in small animal imaging research. We have shown here examples of the value of MRM in studying the normal lung and disease models.

Traditionally proton imaging has been the mainstay of MR, but in the past 10 years, highresolution imaging of gas distribution dynamics using hyperpolarized gas MRI has opened up a new window into functional imaging of the lung. The front-runner in this technology is hyperpolarized ${ }^{3} \mathrm{He}$, which is ideally suited to making exquisite resolution images of gas distribution in the airspaces. ${ }^{3} \mathrm{He}$ MRI is now being routinely applied to functional lung imaging of biological systems ranging from mouse to humans. Hyperpolarized ${ }^{129} \mathrm{Xe}$, which lags technically behind ${ }^{3} \mathrm{He}$, may yet exceed the capabilities of ${ }^{3} \mathrm{He}$ through a powerful combination of its solubility and extraordinary sensitivity to molecular environment. A continued multidisciplinary effort spanning atomic physics, MR physics, spectroscopy, and the involvement of clinicians and biologists could lead this technology to play a central role in non-invasive functional assessment of pulmonary pathologies.

\section{Acknowledgments}

The authors are grateful to Sally Zimney for assistance in preparation of the manuscript and we also thank our many colleagues in the Center for In Vivo Microscopy (CIVM) for their contributions. The Duke CIVM is an NIH NCRR/ 
NCI National Biomedical Technology Resource Center (P41 RR005959/R24 CA092656), with additional support from NIH/NHLBI (R01 HL055348) and GEMI Fund 2005.

\section{References}

Albert MS, Cates GD, Driehuys B, Happer W, Saam B, Springer CS, Wishnia A. Biological magneticresonance imaging using laser polarized ${ }^{129}$ Xe. Nature 1994;370:199-201. [PubMed: 8028666]

Albert MS, Schepkin VD, Budinger TF. Measurement of 129Xe T1 in blood to explore the feasibility of hyperpolarized 129Xe MRI. J Comput Assist Tomogr 1995;19:975-8. [PubMed: 8537536]

Beckmann N, Tigani B, Mazzoni L, Fozard JR. MRI of lung parenchyma in rats and mice using a gradientecho sequence. NMR Biomed 2001;14:297-306. [PubMed: 11477650]

Beckmann N, Tigani B, Mazzoni L, Fozard JR. Techniques: Magnetic resonance imaging of the lung provides potential for noninvasive preclinical evaluation of drugs. Trends Pharmacol Sci 2003;24:550 4. [PubMed: 14559408]

Black RD, Middleton HL, Cates GD, Cofer GP, Driehuys B, Happer W, Hedlund LW, Johnson GA, Shattuck MD, Swartz JC. In vivo He-3 MR images of guinea pig lungs. Radiology 1996;199:867-70. [PubMed: 8638019]

Brau ACS, Hedlund LW, Johnson GA. Cine magnetic resonance microscopy of the rat heart using cardiorespiratory-synchronous projection reconstruction. J Magn Reson Imag 2004;20:31-8.

Bushberg, JT.; Seibert, JA.; Leidholdt, EM.; Boone, JM. The Essential Physics of Medical Imaging. Vol. 2nd. Williams and Wilkins; Baltimore: 2001.

Chen BT, Brau AC, Johnson GA. Measurement of regional lung function in rats using hyperpolarized ${ }^{3}$ helium dynamic MRI. Magn Reson Med 2003;49:78-88. [PubMed: 12509822]

Chen BT, Yordanov AT, Johnson GA. Ventilation-synchronous magnetic resonance microscopy of pulmonary structure and ventilation in mice. Magn Reson Med 2005;53:69-75. [PubMed: 15690504]

Chen XJ, Hedlund LW, Moller HE, Chawla MS, Maronpot RR, Johnson GA. Detection of emphysema in rat lungs by using magnetic resonance measurements of He-3 diffusion. Proc Natl Acad Sci USA 2000;97:11478-81. [PubMed: 11027348]

Chen XJ, Moller HE, Chawla MS, Cofer GP, Driehuys B, Hedlund LW, MacFall JR, Johnson GA. Spatially resolved measurements of hyperpolarized gas properties in the lung in vivo. Part II: T*(2). Magn Reson Med 1999;42:729-37. [PubMed: 10502762]

Cherubini A, Bifone A. Hyperpolarised xenon in biology. Prog Nucl Magn Reson Spectrosc 2003;42:130.

Deninger AJ, Eberle B, Ebert M, Grossmann T, Heil W, Kauczor HU, Lauer L, Markstaller K, Otten E, Schmiedeskamp J, Schreiber W, Surkau R, Thelen M, Weiler N. Quantification of regional intrapulmonary oxygen partial pressure evolution during apnea by He-3 MRI. J Magn Reson 1999;141:207-16. [PubMed: 10579944]

Donnelly LF, MacFall JR, McAdams HP, Majure JM, Smith J, Frush DP, Bogorad P, Charles HC, Ravin CE. Cystic fibrosis: combined hyperpolarized $3 \mathrm{He}$-enhanced and conventional proton MR imaging in the lung- Preliminary observations. Radiology 1999;212:885-889. [PubMed: 10478261]

Driehuys B. Towards molecular imaging with Xenon MRI. Science 2006;314:432-3. [PubMed: 17053138]

Driehuys B, Cofer GP, Pollaro J, Boslego J, Hedlund LW, Johnson GA. Imaging alveolar capillary gas transfer using hyperpolarized 129Xe MRI. Proc Natl Acad Sci USA 2006a;103:18278-83. [PubMed: 17101964]

Driehuys B, Cofer GP, Pollaro J, Walker JK, Schwartz DA, Johnson GA. ${ }^{3}$ He imaging of methacholine challenge in mouse models of asthma. 14th Annual Scientific Meeting, ISMRM 2006b:1347.

Dugas JP, Garbow JR, Kobayashi DK, Conradi MS. Hyperpolarized He-3 MRI of mouse lung. Magn Reson Med 2004;52:1310-17. [PubMed: 15562478]

Ebert M, Grossmann T, Heil W, Otten WE, Surkau R, Leduc M, Bachert P, Knopp MV, Schad LR, Thelen M. Nuclear magnetic resonance imaging with hyperpolarized helium-3. Lancet 1996;347:1297-9. [PubMed: 8622506] 
Fain SB, Panth SR, Evans MD, Wentland AL, Holmes JH, Korosec FR, O'Brien MJ, Fountaine H, Grist TM. Early emphysematous changes in asymptomatic smokers: Detection with He-3 MR imaging. Radiology 2006;239:875-83. [PubMed: 16714465]

Gewalt SL, Glover GH, Hedlund LW, Cofer GP, MacFall JR, Johnson GA. MR microscopy of the rat lung using projection reconstruction. Magn Reson Med 1993;29:99-106. [PubMed: 8419748]

Hedlund LW, Cofer GP, Owen SJ, Johnson GA. MR-compatible ventilator for small animals: computercontrolled ventilation for proton and noble gas imaging. Magn Reson Imag 2000;18:753-9.

Hedlund, LW.; Gewalt, SL.; Cofer, GP.; Johnson, GA. MR microscopy of the lung. In: Cutillo, A., editor. Application of Magnetic Resonance to the Study of the Lung. Futura Press; Mt. Kisco, NY: 1996. p. 401-15.

Hedlund LW, Johnson GA. Morphology of the small animal lung using MR microscopy. Proc Am Thorac Soc 2005;2:481-3. [PubMed: 16352752]

Hornak, JP. The Basics of MRI [Online]. 2006. available at 〈http://www.cis.rit.edu/htbooks/mri/〉

Johnson GA, Cates G, Chen XJ, Cofer GP, Driehuys B, Happer W, Hedlund LW, Saam B, Shattuck MD, Swartz J. Dynamics of magnetization in hyperpolarized gas MRI of the lung. Magn Reson Med 1997;38:66-71. [PubMed: 9211381]

Johnson GA, Cofer GP, Hedlund LW, Maronpot RR, Suddarth SA. Registered H-1 and He-3 magnetic resonance microscopy of the lung. Magn Reson Med 2001;45:365-70. [PubMed: 11241691]

Kadlecek S. Magnetic resonance imaging with polarized gases. Am Sci 2002;90:540-9.

Kadlecek SJ, Emami K, Fischer MC, Ishii M, Yu JS, Woodburn JM, NikKhah M, Vahdat V, Lipson DA, Baumgardner JE, Rizi RR. Imaging physiological parameters with hyperpolarized gas MRI. Prog Nucl Magn Reson Spectrosc 2005;47:187-212.

Koumellis P, van Beek EJR, Woodhouse N, Fichele S, Swift AJ, Paley MNJ, Hill C, Taylor CJ, Wild JM. Quantitative analysis of regional airways obstruction using dynamic hyperpolarized He-3 MRI —Preliminary results in children with cystic fibrosis. J Magn Reson Imaging 2005;22:420-6. [PubMed: 16104046]

Leawoods JC, Yablonskiy DA, Saam B, Gierada DS, Conradi MS. Hyperpolarized He-3 gas production and MR imaging of the lung. Conc Magn Reson 2001;13:277-93.

MacFall JR, Charles HC, Black RD, Middleton H, Swartz JC, Saam B, Driehuys B, Erickson C, Happer W, Cates GD, Johnson GA, Ravin CE. Human lung air spaces: potential for MR imaging with hyperpolarized He-3. Radiology 1996;200:553-8. [PubMed: 8685356]

Mai W, Badea CT, Wheeler CT, Hedlund LW, Johnson GA. Effects of breathing and cardiac motion on spatial resolution in the microscopic imaging of rodents. Magn Reson Med 2005;53:858-65. [PubMed: 15799053]

McAdams HP, Palmer SM, Donnelly LF, Charles HC, Tapson VF, MacFall JR. Hyperpolarized 3Heenhanced MR imaging of lung transplant recipients: preliminary results. Am J Roentgenol 1999;173:955-9. [PubMed: 10511156]

Mentore K, Froh DK, de Lange EE, Brookeman JR, Paget-Brown AO, Altes TA. Hyperpolarized HHe 3 MRI of the lung in cystic fibrosis: Assessment at baseline and after bronchodilator and airway clearance treatment. Acad Radiol 2005;12:1423-9. [PubMed: 16253854]

Moller HE, Chen XJ, Saam B, Hagspiel KD, Johnson GA, Altes TA, de Lange EE, Kauczor HU. MRI of the lungs using hyperpolarized noble gases. Magn Reson Med 2002;47:1029-51. [PubMed: 12111949]

Sakai K, Bilek AM, Oteiza E, Walsworth RL, Balamore D, Jolesz FA, Albert MS. Temporal dynamics of hyperpolarized Xe-129 resonances in living rats. J Magn Reson B 1996;111:300-4. [PubMed: 8661297]

Salerno M, Altes TA, Brookeman JR, de Lange EE, Mugler JP. Dynamic spiral MRI of pulmonary gas flow using hyperpolarized He-3: Preliminary studies in healthy and diseased lungs. Magn Reson Med 2001a;46:667-77. [PubMed: 11590642]

Salerno M, Altes TA, Mugler JP, Nakatsu M, Hatabu H, DeLange EE. Hyperpolarized noble gas MR imaging of the lung: potential clinical applications. Eur J Radiol 2001b;40:33-44. [PubMed: 11673006] 
Salerno M, de Lange EE, Altes TA, Truwit JD, Brookeman JR, Mugler JP. Emphysema: hyperpolarized helium 3 diffusion MR imaging of the lungs compared with spirometric indexes-Initial experience. Radiology 2002;222:252-60. [PubMed: 11756734]

Samee S, Altes T, Powers P, de Lange EE, Knight-Scott J, Rakes G, Mugler JP, Ciambotti JM, Alford BA, Brookeman JR, Platts-Mills TAE. Imaging the lungs in asthmatic patients by using hyperpolarized helium-3 magnetic resonance: Assessment of response to methacholine and exercise challenge. J Allergy Clin Immunol 2003;111:1205-11. [PubMed: 12789218]

Samee S, Altes T, Powers PL, Rakes G, Knight-Scott J, Mata J, Christopher JM, Delange EE, Alford BA, Brookeman J, Platts-Mills TA. Identification of reversible ventilation defects in the lungs of asthmatic patients using hyperpolarized helium-3 MR. J Allergy Clin Immunol 2001;107:757.

Schroder L, Lowery TJ, Hilty C, Wemmer DE, Pines A. Molecular imaging using a targeted magnetic resonance hyperpolarized biosensor. Science 2006;314:446-9. [PubMed: 17053143]

Schuster DP, Kovacs A, Garbow J, Piwnica-Worms D. Recent advances in imaging the lungs of intact small animals. Am J Respir Cell Mol Biol 2004;30:129-38. [PubMed: 14729505]

van Beek EJR, Schmiedeskamp J, Wild JM, Paley MNJ, Filbir F, Fichele S, Knitz F, Mills GH, Woodhouse N, Swift A, Heil W, Wolf M, Otten E. Hyperpolarized 3-helium MR imaging of the lungs: testing the concept of a central production facility. Eur Radiol 2003;13:2583-6. [PubMed: 14556034]

van Beek EJR, Wild JM, Kauczor HU, Schreiber W, Mugler JP, de Lange EE. Functional MRI of the lung using hyperpolarized 3-helium gas. J Magn Reson Imag 2004;20:540-54.

Viallon M, Berthezene Y, Callot V, Bourgeois M, Humblot H, Briguet A, Cremillieux Y. Dynamic imaging of hyperpolarized He-3 distribution in rat lungs using interleaved-spiral scans. NMR Biomed 2000;13:207-13. [PubMed: 10867698]

Ward ER, Hedlund LW, Kurylo WC, Wheeler CT, Cofer GP, Dewhirst MW, Marks LB, Vujaskovic Z. Proton and hyperpolarized helium magnetic resonance imaging of radiation-induced lung injury in rats. Int J Radiat Oncol Biol Phys 2004;58:1562-9. [PubMed: 15050337]

\section{Abbreviations}

$3 D$

Three-dimensional

ADC

Apparent diffusion coefficient

COPD

Chronic obstructive pulmonary disease

Gd

Gadolinium

H

Hydrogen

He

Helium

HP

Hyperpolarized

M

Molar (mol/liter)

MIP

Maximum intensity projection

MR

Toxicol Pathol. Author manuscript; available in PMC 2009 September 21. 
Magnetic resonance

MRI

Magnetic resonance imaging

Magnetic resonance microscopy

$\mathbf{O}_{2}$

Oxygen

ppm

Parts per million

$\mathbf{R b}$

Rubidium

RBC

Red blood cell

SNR

Signal-to-noise ratio

$\mathbf{T}$

Tesla

$\mu \mathbf{L}$

Micro liter $\left(\mathrm{mm}^{3}\right)$

$\mathrm{Xe}$

Xenon

Toxicol Pathol. Author manuscript; available in PMC 2009 September 21. 

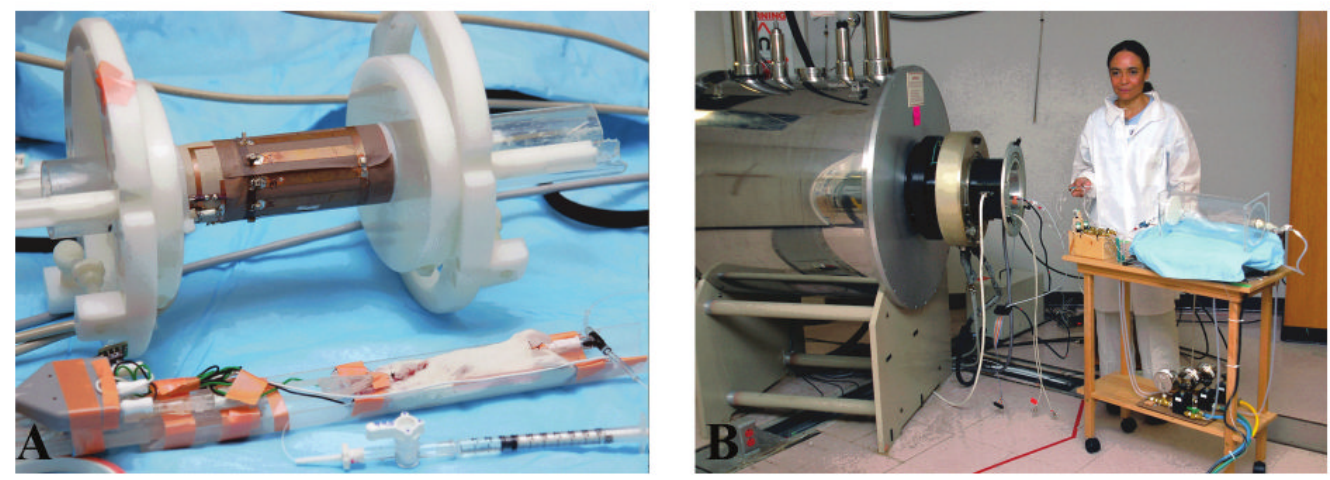

Figure 1.

A. Apparatus for in vivo imaging showing a 23-gram mouse in an imaging cradle with ECG and temperature leads attached, with a 3 -cm inner diameter, dual-frequency imaging $\left({ }^{1} \mathrm{H}-{ }^{3} \mathrm{He}\right)$ MR imaging coil in the background. B. Ventilation system near the bore of the $2 \mathrm{~T}$ MR small animal imaging system, after the mouse and coil have been inserted into the magnet. 


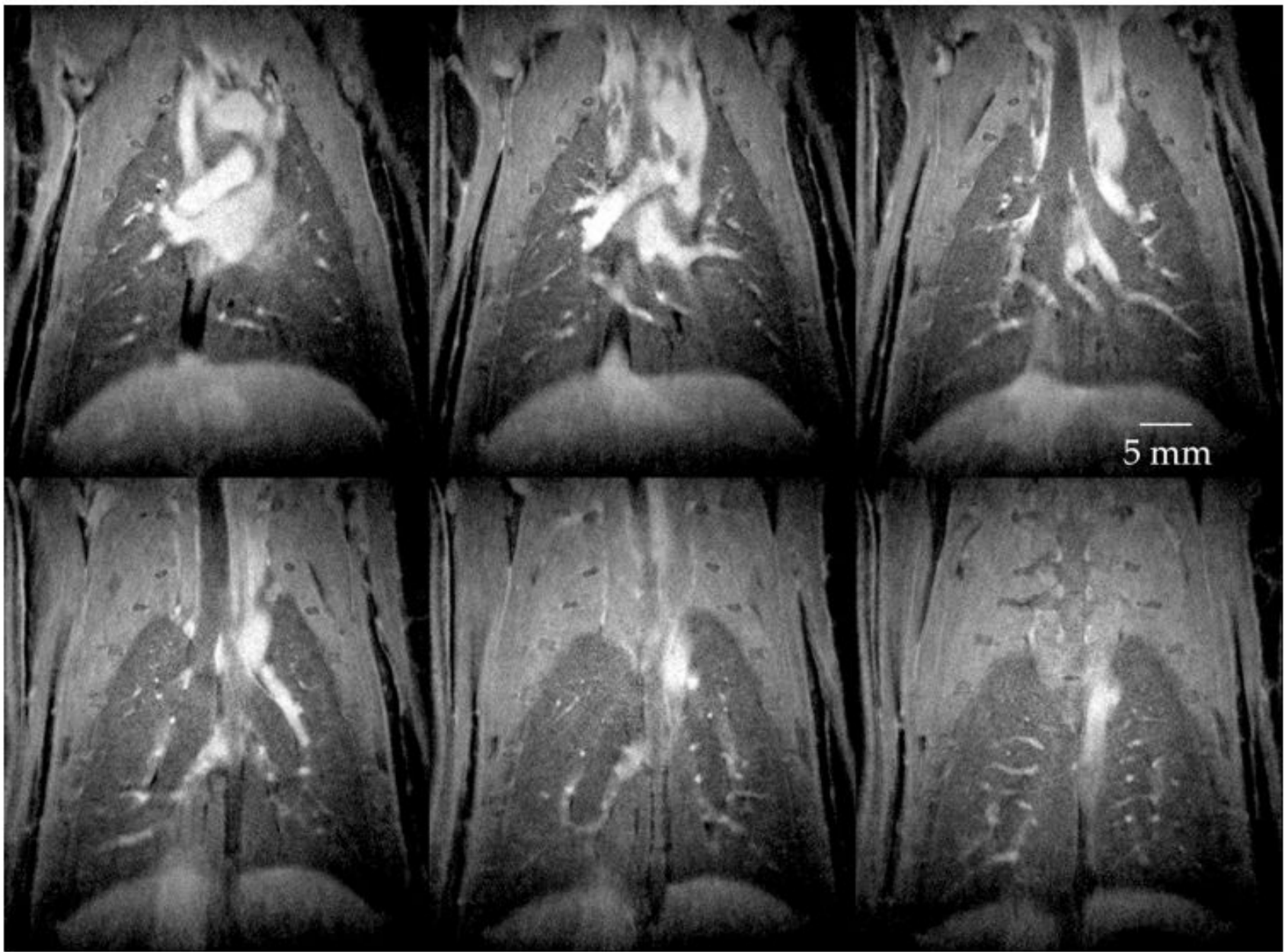

Figure 2.

Example of a high-resolution proton morphologic image of a rat lung using the $2 \mathrm{~T}$ system shown in Figure 1. The parenchyma signal intensity has been enhanced by an infusion of gadolinium chelate $(\mathrm{Gd})$ contrast agent (Magnevist Berlex Imaging, Wayne, NJ). These 6 slices are taken from an isotropic dataset with resolution of $117 \times 117 \times 117 \mu \mathrm{m}^{3}$. Reprinted with permission from (Johnson et al., 2001). 


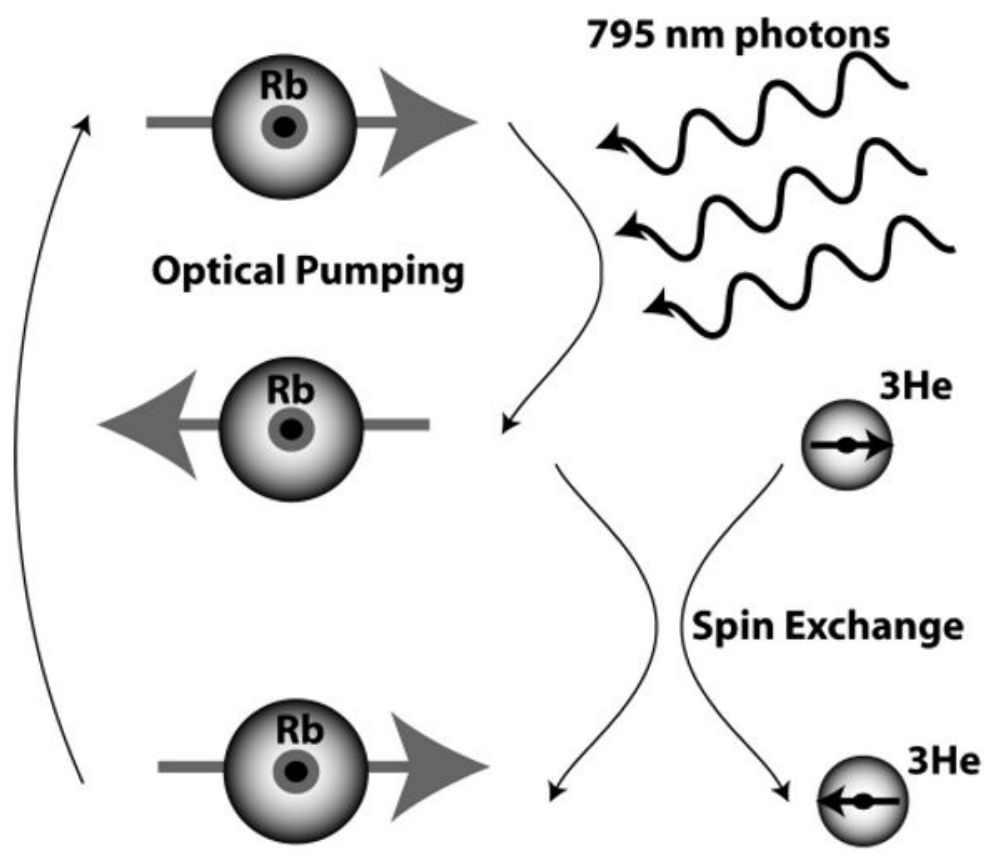

Figure 3.

Schematic representation of hyperpolarization by optical pumping and spin exchange. ${ }^{3} \mathrm{He}$ and ${ }^{129} \mathrm{Xe}$ nuclei acquire a high degree of nuclear magnetic alignment through angular momentum transfer from laser photons to vaporized Rubidium $(\mathrm{Rb})$ and then spin exchange with ${ }^{3} \mathrm{He} /{ }^{129} \mathrm{Xe}$. In this hyperpolarized state, these gases enhance the MRI signal by roughly $10^{5}$, more than enough to overcome the low density and enable the gases to be imaged with exquisite resolution. 


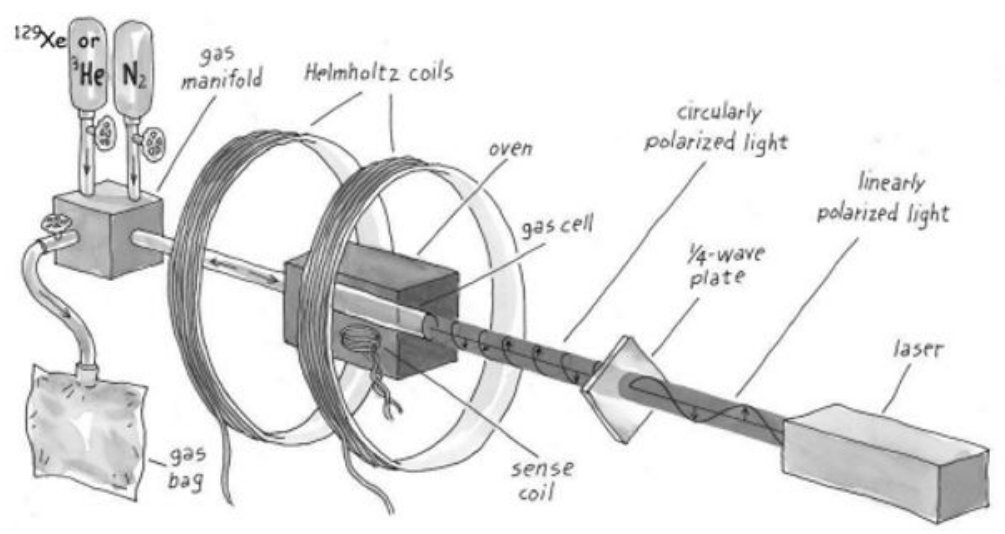

Figure 4.

A schematic of a gas polarizer. Laser light is circularly polarized so that all photons carry +1 quantum of angular momentum. The laser light is absorbed by an Rb vapor in the optical gas cell, thus aligning the valence electrons of all the $\mathrm{Rb}$ atoms with the weak magnetic field provided by the Helmholtz coils. Collisions between polarized $\mathrm{Rb}$ atoms and ${ }^{3} \mathrm{He}$ or ${ }^{129} \mathrm{Xe}$ atoms transfer the alignment to the ${ }^{3} \mathrm{He}$ or ${ }^{129} \mathrm{Xe}$ nuclei, as illustrated in Figure 3. The oven elevates the gas cell temperature to about $200^{\circ} \mathrm{C}$ to vaporize the $\mathrm{Rb}$. The sense coil monitors the extent of gas polarization. (Reprinted with permission from (Kadlecek et al., 2002). 


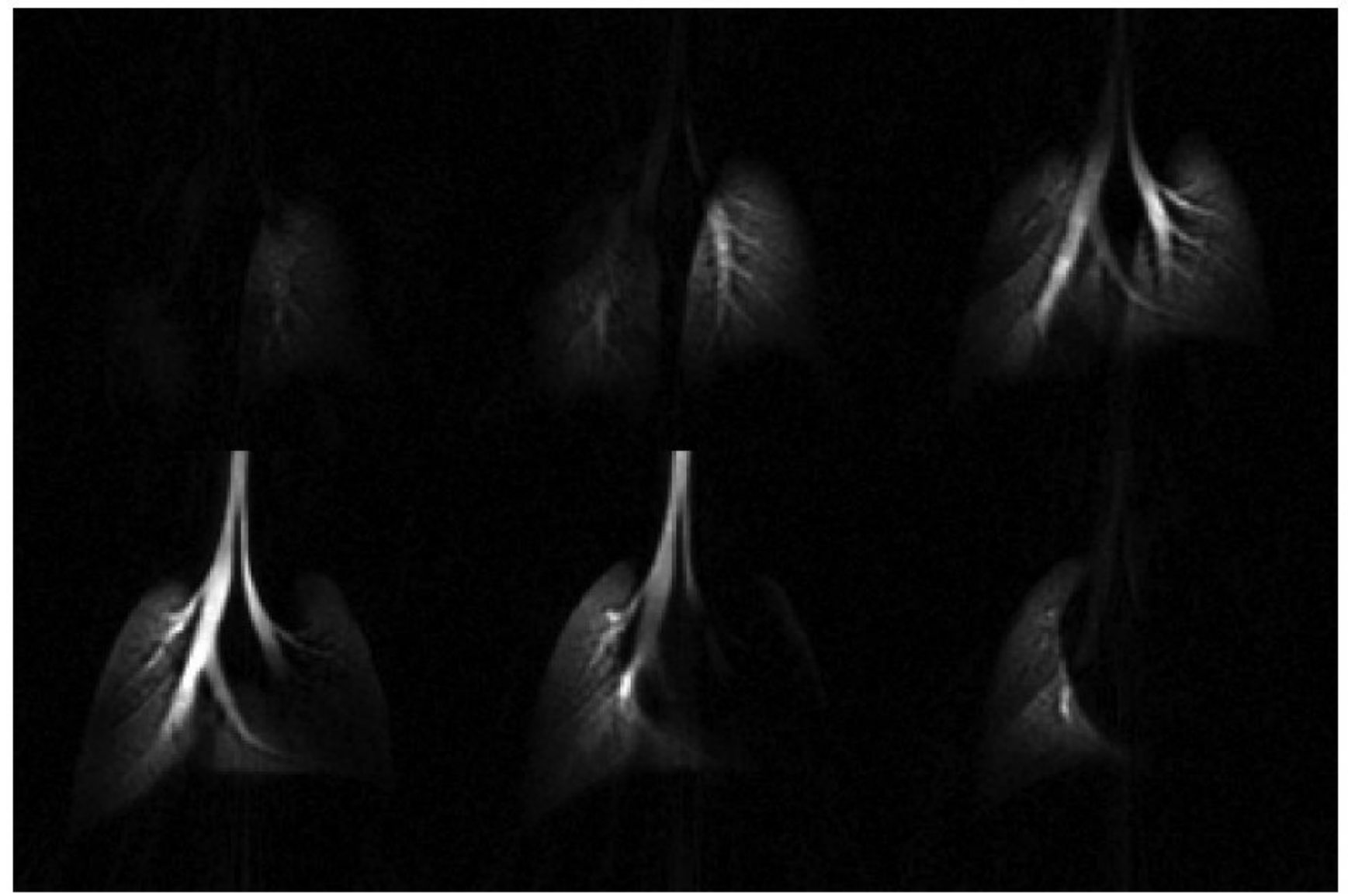

Figure 5.

$3 \mathrm{D}{ }^{3} \mathrm{He}$ MR images of a 25 -gram mouse. The resolution is $125 \times 125 \times 1000 \mu \mathrm{m}$. The image required 5.8 minutes to acquire and consumed $92 \mathrm{ml}$ of hyperpolarized ${ }^{3} \mathrm{He}$. 


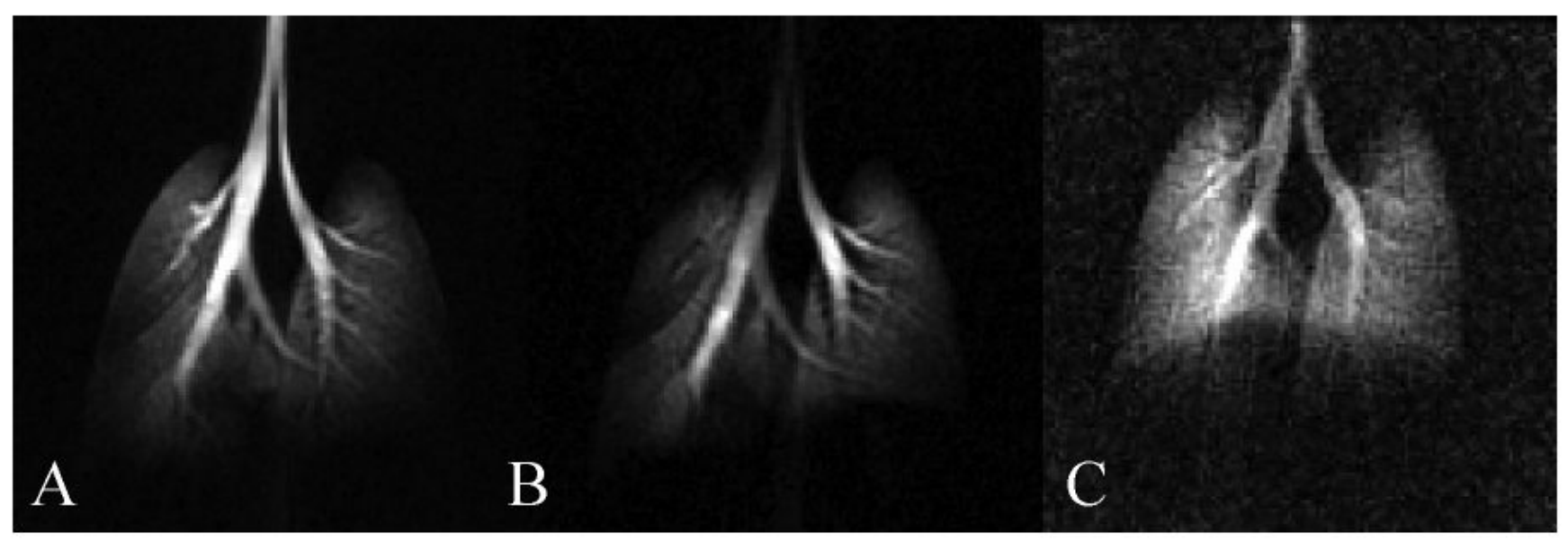

Figure 6.

These ${ }^{3} \mathrm{He}$ images illustrate different representations and image acquisition strategies. (A) Maximum intensity projection (MIP) of all coronal slices in the three-dimensional dataset shown in Figure 5. This MIP representation acquired in 5.8 minutes more clearly shows major lobar airways. (B) A single central slice from the same dataset as in Figure 4. (C) A full dorsoventral projection image from a different mouse required only 12 seconds to acquire and consumed only $2 \mathrm{ml}$ of $\mathrm{HP}{ }^{3} \mathrm{He}$. However, this image shows less detail of the airways. These images illustrate the trade-offs that are made in image acquisition and temporal resolution to derive the desired information. 


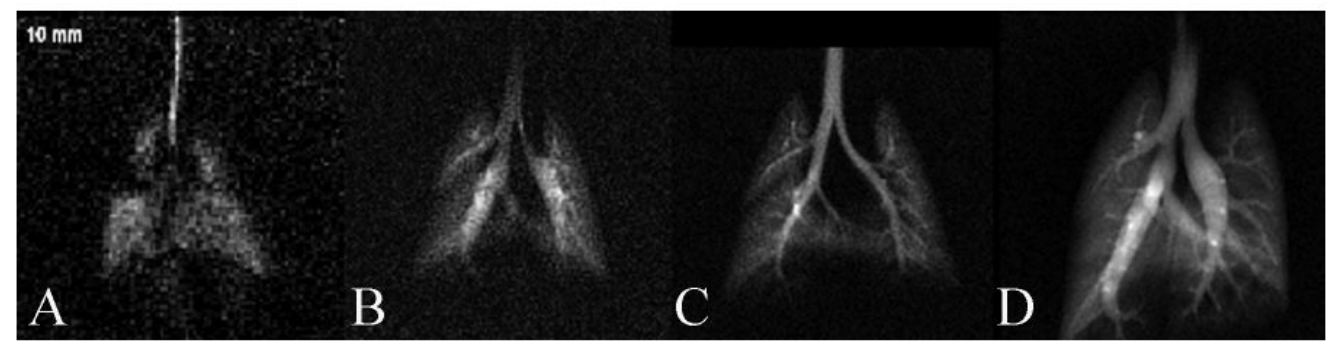

Figure 7.

The progress in xenon ventilation imaging in the rat. (A) One of the first ${ }^{129} \mathrm{Xe}$ images made in this laboratory in 1998 with $0.84 \times 0.84 \mathrm{~mm}^{2}$ resolution and SNR of about 3 . (B-D) show progressively better image quality as polarization, gas delivery technology, and MR acquisition strategies have been improved. Our current standard ${ }^{129} \mathrm{Xe}$ image (D) with resolution of 0.31 $\times 0.31 \mathrm{~mm}^{2}$ and an SNR of about 20. Continued progress in these areas should lead to even further improvement in image quality. 

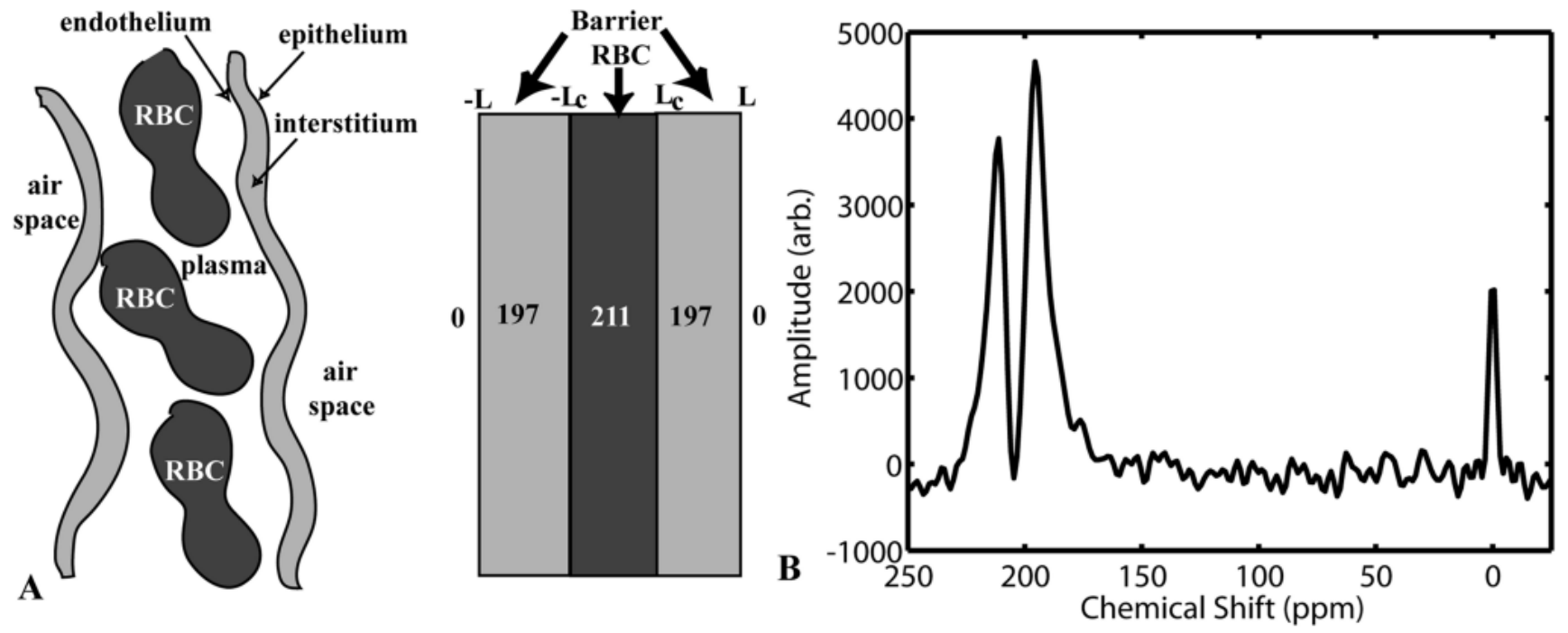

Figure 8.

(A) A schematic representation of the different molecular environments experienced by ${ }^{129} \mathrm{Xe}$ in the lung (left) and (right) schematic summary of functional compartments: air space (0), barrier (197) (alveolar epithelium, interstitium, capilliary endothelium, plasma), and red blood cells (RBCs) (211). (B) A spectrum of ${ }^{129} \mathrm{Xe}$ in the rat lung. The spectrum exhibits 3 distinct resonances at $211 \mathrm{ppm}$ (RBC), $197 \mathrm{ppm}$ (barrier space), and $0 \mathrm{ppm}$ (air space). The ability to discriminate ${ }^{129} \mathrm{Xe}$ in these distinct environments creates a unique tool to measure the transfer dynamics of ${ }^{129} \mathrm{Xe}$ among these various compartments, thereby supporting measurement of micron-scale thickening of the alveolar-capillary barrier, such as may be present in inflammation or fibrosis. 


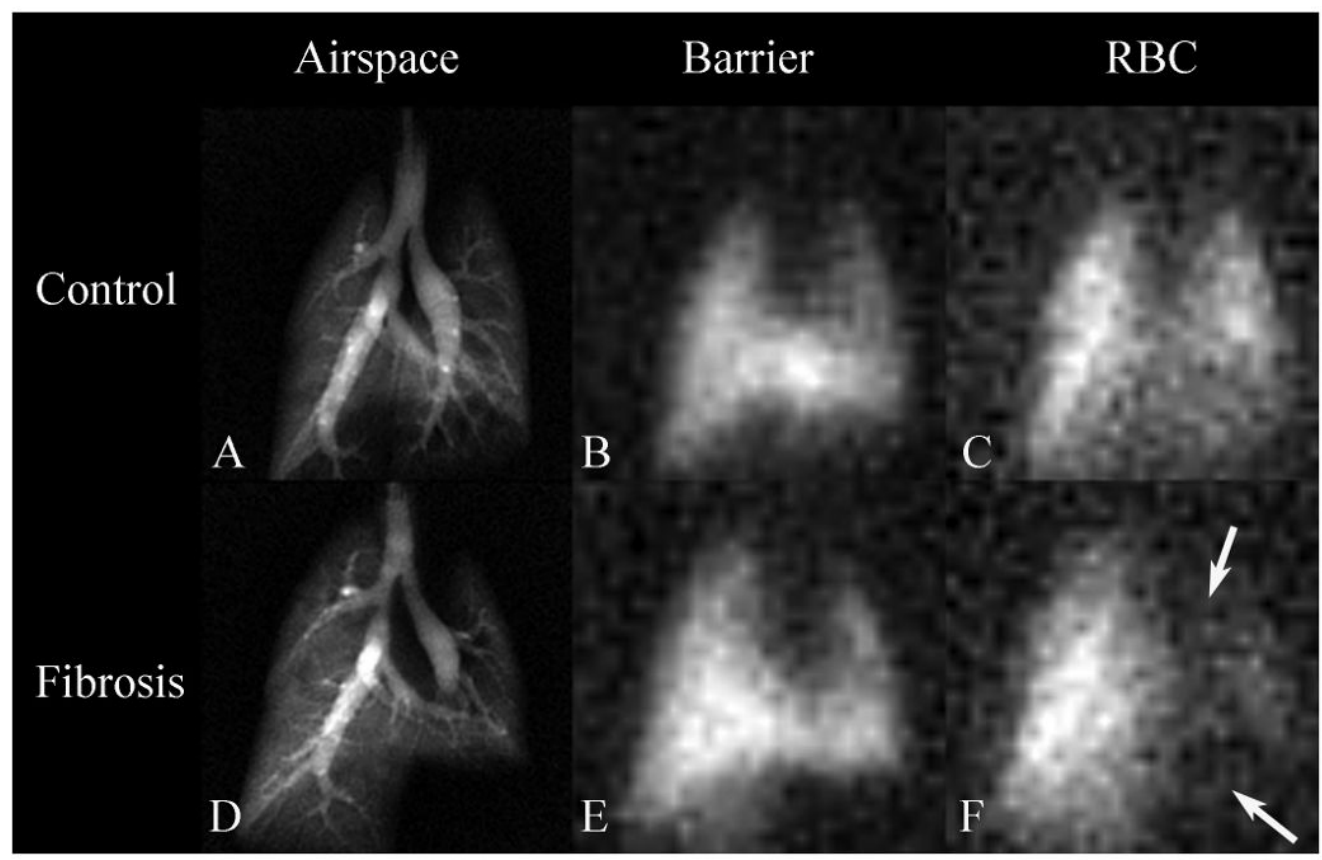

Figure 9.

${ }^{129} \mathrm{Xe}$ images in three different pulmonary compartments-gas space $(\mathrm{A}, \mathrm{D})$, barrier $(\mathrm{B}, \mathrm{E})$, and $\mathrm{RBC}(\mathrm{C}, \mathrm{F})$. The top panels $(\mathrm{A}-\mathrm{C})$ depict a control rat. The bottom panels $(\mathrm{D}-\mathrm{F})$ show a rat with left lung fibrosis from a unilateral instillation of bleomycin. In the fibrotic lung, there is full absorption into the "barrier" tissue space (E) and a dramatic lack of ${ }^{129} \mathrm{Xe}$ signal in the RBC compartment (arrows) (F). We hypothesize that the lack of RBC signal is due to the increased diffusion time required for ${ }^{129} \mathrm{Xe}$ to traverse the thickened blood-gas barrier. 